\title{
Activation of the cGMP/cGKI signalling pathway in primary murine VSMCs accelerates growth
}

\author{
Pascal Weinmeister*1, Robert Lukowski ${ }^{1}$, Claudia Traidl-Hoffmann², \\ Stefan Linder ${ }^{3}$, Franz Hofmann ${ }^{1}$ and Robert Feil ${ }^{4}$
}

\author{
Address: ${ }^{1}$ Institut für Pharmakologie und Toxikologie, TU München, Germany, ${ }^{2}$ Zentrum für Allergie und Umwelt, TU München, Germany, \\ ${ }^{3}$ Institut für Prophylaxe und Epidemiologie der Kreislaufkrankheiten, LMU München, Germany and ${ }^{4}$ Interfakultäres Institut für Biochemie, \\ Universität Tübingen, Germany \\ Email: Pascal Weinmeister* - weinmeister@ipt.med.tu-muenchen.de \\ * Corresponding author
}

\author{
from $3^{\text {rd }}$ International Conference on cGMP Generators, Effectors and Therapeutic Implications \\ Dresden, Germany. 15-17 June 2007 \\ Published: 25 July 2007 \\ BMC Pharmacology 2007, 7(Suppl I):P64 doi:I0.1 186/I47I-2210-7-SI-P64
}

This abstract is available from: http://www.biomedcentral.com/I47I-22I0/7/SI/P64

(c) 2007 Weinmeister et al; licensee BioMed Central Ltd.

The endogenous signaling molecule nitric oxide (NO) exerts many of its actions via the cyclic guanosine monophosphate (cGMP)/cGMP-dependent protein kinase type I (cGKI) pathway. Recently, the analysis of an in vivo mouse model for atherosclerosis suggested that activation of cGKI in vascular smooth muscle cells (VSMCs) promotes the phenotypic modulation of medial VSMCs and, thus, vascular lesion formation. In contrast, many in vitro studies demonstrate an anti-proliferative role for cGKI. In the current study, primary and subcultured wild-type and cGKI-deficient VSMCs in response to the membrane permeable cGMP analogue 8-Br-cGMP were compared. In agreement with the common view an anti-proliferative effect of cGKI was found in repeatedly passaged VSMCs (P5-11). In contrast, the analysis of primary VSMCs revealed that activation of cGKI in primary VSMCs strongly promotes growth. Analysing proliferation, apoptosis, cytoskeletal dynamics and various signaling pathways indicated that an increase in cell adhesion is a major mechanism for cGKI-mediated "growth" in primary VSMCs. This pro-adhesive effect of cGKI might be mediated via an inhibition of Rho kinase (ROCK) and enhanced integrin signaling. These processes might contribute to inhibit anoïkis, the programmed cell death induced by the loss of cell/matrix interactions. 